\title{
Telescope Array search for EeV photons and neutrinos
}

\section{Grigory Rubtsov ${ }^{* \dagger}$}

Institute for Nuclear Research of the Russian Academy of Sciences, Moscow, Russia

E-mail: grisha@ms2.inr.ac.ru

\section{Masaki Fukushima}

Institute for Cosmic Ray Research, University of Tokyo, Kashiwa, Chiba, Japan

E-mail: fukushimeicrr.u-tokyo.ac.jp

\section{Dmitri Ivanov}

University of Utah, Department of Physics \& Astronomy and High Energy Astrophysics Institute,

Salt Lake City, Utah, USA

E-mail: dmiivanov@gmail.com

\section{Mikhail Kuznetsov}

Institute for Nuclear Research of the Russian Academy of Sciences, Moscow, Russia

E-mail: mkuzn@ms2.inr.ac.ru

\section{Maxim Piskunov}

Institute for Nuclear Research of the Russian Academy of Sciences, Moscow, Russia

E-mail: maxitg@gmail.com

\section{Gordon Thomson}

University of Utah, Department of Physics \& Astronomy and High Energy Astrophysics Institute,

Salt Lake City, Utah, USA

E-mail: thomsonephysics.utah.edu

\section{Sergey Troitsky}

Institute for Nuclear Research of the Russian Academy of Sciences, Moscow, Russia

E-mail: stems2.inr.ac.ru

\section{Yana Zhezher}

Institute for Nuclear Research of the Russian Academy of Sciences, Moscow, Russia

E-mail: zhezher.yana@physics.msu.ru

We present the searches for photons and neutrinos with the 9 years of data of the Telescope Array surface detector (2008-2017). The both searches employ multivariate analysis with the classifier based on the Boosted Decision Tree. The diffuse flux limits for photons and down-going neutrinos are obtained for the primary energies greater than $1 \mathrm{EeV}$.

35th International Cosmic Ray Conference

10-20 July, 2017

Bexco, Busan, Korea

\footnotetext{
* Speaker.

${ }^{\dagger}$ for the Telescope Array collaboration
} 


\section{Introduction}

Telescope Array (TA) experiment [1,2] is a hybrid detector operating in Utah, USA. TA consists of a surface detector array of 507 plastic scintillators with $1.2 \mathrm{~km}$ spacing covering $700 \mathrm{~km}^{2}$ area and three fluorescence detectors. The purpose of this Talk is to present the limits on diffuse photon and neutrino flux with energies greater than $10^{18} \mathrm{eV}$ based on the 9 years of surface detector operation.

Several limits on the ultra-high-energy diffuse photon flux have been set by independent experiments, including Haverah Park, AGASA, Yakutsk, Pierre Auger and Telescope Array observatories $[3,4,5,6,7,8,9,10,11,12,13]$, but no evidence for primary photons found at present. The upper limit on a photon flux from Southern hemisphere point sources is set by the Pierre Auger Observatory [14]. Photon flux limits may be used to constrain the parameters of top-down models [15] and the properties of the astrophysical sources and their evolution in the scenario of Greisen-Zatsepin-Kuzmin [16, 17] cut-off. Moreover, the results of the photon search severely constrain the parameters of Lorentz invariance violation at Planck scale [18, 19, 20, 21, 22]. Finally, photons with energies above $\sim 10^{18} \mathrm{eV}$ might be responsible for CR events correlated with BL Lac type objects on the angular scale significantly smaller than the expected deflection of protons in cosmic magnetic fields and thus suggesting neutral primaries [23, 24] (see Ref. [25] for a particular mechanism).

Ultra-high energy neutrinos may be generated by the decay of the charged pions produced as secondaries in GZK process [26], by beta-decay of unstable ultra-high energy nuclei or by some mechanism related to the new physics. Neutrino flux at ultra-high energies is constrained by air shower [27, 28, 29, 30], ice shower [31] and radio Cherenkov [32, 33, 34, 35, 36, 37] experiments (see [38] for a review). At lower $(\mathrm{PeV})$ energies, neutrinos of astrophysical origin are discovered by IceCube [39].

\section{Data set and simulations}

We use Telescope Array surface detector data set covering nine years of observation from 2008-05-11 to 2017-05-11. Surface detector has been collecting data for more than $95 \%$ of time during that period [40].

Air showers induced by primary photons differ significantly from the hadron-induced events (see e.g. [41] for a review). The Telescope Array surface detectors contain plastic scintillators of $3 \mathrm{~m}^{2}$ area which detect both muon and electromagnetic components of the extensive air shower and therefore are sensitive to showers induced by primary photons (see Ref. [42] for discussion).

The results of this Talk employ Monte-Carlo simulations reproducing the 9 years of observations at the TA SD. The proton induced simulated shower set is used as a background while the photon or neutrino set as a signal. We produce simulated events by CORSIKA [43] with EGS4 [44] model for electromagnetic interactions, PRESHOWER code [45] for interactions of photons in geomagnetic field, QGSJET II-03 [46] and FLUKA [47] for high and low energy hadronic interactions. The showers are simulated with thinning and the dethinning procedure is used to recover small scale structure of the shower fluctuations [48]. 
Detector response is accounted for by using look-up tables simulated with GEANT4 [49]. Real-time array configuration and detector calibration information are used for each simulated event. Monte-Carlo (MC) events are produced in the same format as real events and the analysis procedures are applied in the same way to both [48].

\section{Reconstruction and observables}

We reconstruct each event with a joint fit of the geometry and lateral distribution function (LDF) and determine Linsley shower front curvature parameter " $a$ " along with the arrival direction, core location and signal density at 800 meters $\mathscr{S} \equiv S_{800}$. One and the same reconstruction procedure is applied to both data and MC events.

For each real event "i" we estimate the energy of hypothetical photon primary $E_{\gamma}^{i}=$ $E_{\gamma}\left(\mathscr{S}^{i}, \theta^{i}, \phi^{i}\right)$, i.e. the average energy of the primary photon, inducing the shower with the same arrival direction and $\mathscr{S}$. The look-up table for $E_{\gamma}(\mathscr{S}, \theta, \phi)$ is built using photon MC set.

The following cuts are applied to both data and MC events:

(a) Zenith angle cut: $0^{\circ}<\theta<60^{\circ}$ for photon search and $45^{\circ}<\theta<90^{\circ}$ for neutrino search;

(b) The number of detectors triggered is 7 or more for photon search and 5 or more for neutrino search;

(c) Shower core is inside the array boundary with the distance to the boundary larger than 1200 meters;

(d) Joint fit quality cut, $\chi^{2} /$ d.o.f. $<5$;

(e) $E_{\gamma}\left(S_{800}^{i}, \theta^{i}, \phi^{i}\right)>E_{0} \mathrm{eV}$ for photon search.

In addition to these cuts, the events which are the part of the millisecond-time event bursts [50] are removed from the data set. There is an evidence that these events are caused by lightnings [50].

Reconstruction of each data and MC event results in a set of the following observables used for construction of a multivariate classification method:

1. Zenith angle, $\theta$;

2. Signal density at $800 \mathrm{~m}$ from the shower core, $S(800)$;

3. Linsley front curvature parameter, $a$;

4. Area-over-peak (AoP) of the signal at $1200 \mathrm{~m} \mathrm{[51];}$

5. AoP slope parameter [52];

6. Number of detectors hit;

7. Number of detectors excluded from the fit of the shower front;

8. $\chi^{2} /$ d.o.f.; 


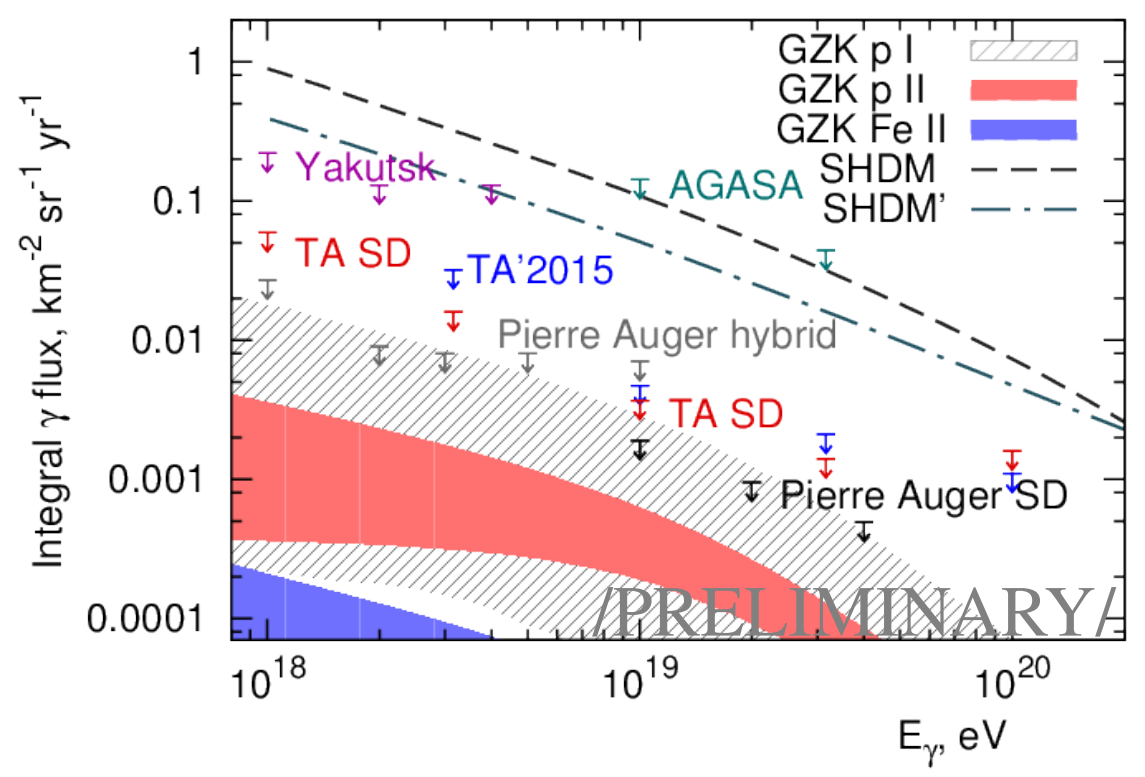

Figure 1: Photon flux limit presented in this Talk (TA SD) compared with the results from AGASA [4], Pierre Auger Observatory SD [10] and hybrid data [11], Yakutsk [6] and TA ICRC'2015 (TA'15) [13] and the predictions of certain models [38].

9. $S_{b}$ parameter for $b=3[53]$;

10. $S_{b}$ parameter for $b=4.5$;

11. The sum of signals of all detectors of the event;

12. An average asymmetry of signal at upper and lower layers of the detectors;

13. Total number of peaks over both upper and lower layers of all detectors hit. To suppress accidental peaks as a result of FADC noise we define a peak as a time bin with a signal above 0.2 VEM which is higher than a signal of 3 preceding and 3 consequent time bins.

14. Number of peaks for the detector with the largest signal;

15. Total number of peaks present in the upper layer and not in lower;

16. Total number of peaks present in the lower layer and not in upper;

\subsection{Method}

The analysis is based on the proton-photon classification procedure using the method of boosted decision trees (BDT). Following the analyses of [14, 52] we use the TMVA package [54] for ROOT as an implementation of the method. The decision forest is constructed using 15 observable parameters listed in Section. 3. The BDT is trained using the proton Monte-Carlo set as a background and the photon or all-flavor down-going neutrino Monte-Carlo as a signal. The MonteCarlo set is split into three equal parts: (I) for training the classifier, (II) for cut optimization, (III) 
for exposure estimate. For the photon search the classifier is built independently for each energy range of interest $E>E_{0}$, where $E_{0}$ takes values of $10^{18.0}, 10^{18.5}, 10^{19.0}, 10^{19.5}$ and $10^{20.0} \mathrm{eV}$.

The result of the BDT classifier is a single parameter $\xi^{i}$ for each event " $\mathrm{i}$ " which has a bounder range by definition $-1 \leq \xi^{i} \leq 1$. The $\xi$-parameter is finally used for one-parametric composition analysis.

The photon or neutrino candidates are selected with the zenith angle dependent cut on $\xi$

$$
\xi>\xi_{\text {cut }}(\theta)
$$

The cut function is approximated as a quadratic polynomial of $\theta$. The cut is optimized with the part II of MC with the merit factor defined as an average photon flux upper limit in the case of null-hypothesis: all events in the data set are protons.

\section{Photon search results}

Geometrical exposure for the considered SD observation period with $0^{\circ}<\theta<60^{\circ}$ and boundary cut is given by

$$
A_{\text {geom }}=12063 \mathrm{~km}^{2} \mathrm{sr} \mathrm{yr} .
$$

The effective exposure after the cuts $A_{e f f}^{\gamma}$ is given in Table 1.

\begin{tabular}{|c|c|c|c|}
\hline$E_{0}$ & quality cuts (a)-(e) & $\xi$-cut & $A_{\text {eff }}^{\gamma} \mathrm{km}^{2}$ sryr \\
\hline $10^{18.0}$ & $6.8 \%$ & $6.5 \%$ & $\mathbf{5 3}$ \\
\hline $10^{18.5}$ & $20.1 \%$ & $7.9 \%$ & $\mathbf{1 9 2}$ \\
\hline $10^{19.0}$ & $43.8 \%$ & $16.0 \%$ & $\mathbf{8 4 6}$ \\
\hline $10^{19.5}$ & $52.1 \%$ & $34.0 \%$ & $\mathbf{2 1 3 8}$ \\
\hline $10^{20.0}$ & $64.7 \%$ & $55.3 \%$ & $\mathbf{4 3 1 7}$ \\
\hline
\end{tabular}

Table 1: Contribution of the cuts to an effective exposure at energy ranges of interest. The value represents a ratio of the exposure after the given cut to the exposure before cut.

After the cuts there are no photon candidates found in the data set for $E_{0}=10^{18.0}, 10^{18.5}, 10^{19.0}$ and $10^{19.5} \mathrm{eV}$. There are two candidates for $E_{0}=10^{20.0} \mathrm{eV}$. An upper limit on a mathematical expectation of number of photons is determined following Ref. [55]. The flux upper limits follow from the relation

$$
\bar{n}_{\gamma}=F_{\gamma} A_{e f f}^{\gamma} .
$$

The resulting 95\% CL photon diffuse flux upper limits are summarized in Table 2 and are compared to the results of other experiments in Figure 1.

The two photon candidate events have the timestamps 2013-08-27 22:38:37.991329 and 201408-23 02:39:15.953765. It turned out that these timestamps are within $1 \mathrm{~ms}$ of lightning events detected by the National Lightning Detection Network [56] at the location of the Telescope Array. The further study of the possible terrestrial origin of the photon candidate events is in progress.

The implementation of the multivariate classifier permits to search for the photon-induced showers in the wide range of zenith angles $\left(0^{\circ}<\theta<60^{\circ}\right)$ with the enhanced effective exposure. 


\begin{tabular}{|c|l|l|l|l|l|}
\hline & \multicolumn{5}{|c|}{$E_{0}, \mathrm{eV}$} \\
& $10^{18.0}$ & $10^{18.5}$ & $10^{19.0}$ & $10^{19.5}$ & $10^{20.0}$ \\
\hline$\gamma$ candidates & 0 & 0 & 0 & 0 & 2 \\
\hline $\bar{n}_{\gamma}<$ & 3.09 & 3.09 & 3.09 & 3.09 & 6.72 \\
\hline$A_{\text {eff }}^{\gamma}$ & 53 & 192 & 846 & 2138 & 4317 \\
\hline$F_{\gamma}<$ & 0.059 & 0.016 & 0.0037 & 0.0014 & 0.0016 \\
\hline
\end{tabular}

Table 2: Preliminary 95\% CL upper limits on the number of photons in the data set $\bar{n}_{\gamma}$ and on the photon flux $F_{\gamma}\left(\mathrm{km}^{-2} \mathrm{yr}^{-1} \mathrm{sr}^{-1}\right)$.

\section{Neutrino search results}

Geometrical exposure for $45^{\circ}<\theta<90^{\circ}$ is $A_{\text {geom }}^{v}=8042 \mathrm{~km}^{2}$ sr yr.. The effective exposure for the down-going neutrino with respect to the single flavor neutrino flux is $A_{\text {eff }}^{v}=$ $5.7 \cdot 10^{-3} \mathrm{~km}^{2} \mathrm{sr} \mathrm{yr}$.. This value accounts for neutrino interaction probability in the atmosphere, trigger, reconstruction and cut efficiencies.

There are no neutrino candidates in the data set and hence using Ref. [55] we arrive at the following single flavor diffuse neutrino flux limit for $E>10^{18} \mathrm{eV}$ :

$$
E^{2} f_{v}<1.4 \times 10^{-6} \mathrm{GeV} \mathrm{cm}^{-2} \mathrm{~s}^{-1} \mathrm{sr}^{-1} \quad(90 \% \text { C.L. })
$$

\section{Acknowledgment}

The Telescope Array experiment is supported by the Japan Society for the Promotion of Science through Grants-in-Aid for Scientific Research on Specially Promoted Research (21000002) "Extreme Phenomena in the Universe Explored by Highest Energy Cosmic Rays" and for Scientific Research (19104006), and the Inter-University Research Program of the Institute for Cosmic Ray Research; by the U.S. National Science Foundation awards PHY-0307098, PHY-0601915, PHY-0649681, PHY-0703893, PHY-0758342, PHY-0848320, PHY-1069280, PHY-1069286, PHY-1404495 and PHY-1404502; by the National Research Foundation of Korea (2015R1A2A1A01006870, 2015R1A2A1A15055344, 2016R1A5A1013277, 20070093860, 2016R1A2B4014967); by the Russian Academy of Sciences, RFBR grant 16-02-00962a (INR), IISN project No. 4.4502.13, and Belgian Science Policy under IUAP VII/37 (ULB). The foundations of Dr. Ezekiel R. and Edna Wattis Dumke, Willard L. Eccles, and George S. and Dolores Doré Eccles all helped with generous donations. The State of Utah supported the project through its Economic Development Board, and the University of Utah through the Office of the Vice President for Research. The experimental site became available through the cooperation of the Utah School and Institutional Trust Lands Administration (SITLA), U.S. Bureau of Land Management (BLM), and the U.S. Air Force. We appreciate the assistance of the State of Utah and Fillmore offices of the BLM in crafting the Plan of Development for the site. We also wish to thank the people and the officials of Millard County, Utah for their steadfast and warm support. We gratefully acknowledge the contributions from the technical staffs of our home institutions. An allocation of computer time from the Center for High Performance Computing at the University of Utah is gratefully acknowledged. The cluster of the Theoretical Division of INR RAS was used for the numerical part of the work. 


\section{References}

[1] T. Abu-Zayyad et al. [Telescope Array Collaboration], Nucl. Instrum. Meth. A 689, 87 (2012).

[2] H. Tokuno et al. [Telescope Array Collaboration] Nucl. Instrum. Meth. A 676, 54 (2012).

[3] M. Ave, J. A. Hinton, R. A. Vazquez, A. A. Watson and E. Zas, Phys. Rev. Lett. 85, 2244 (2000).

[4] K. Shinozaki et al., Astrophys. J. 571, L117 (2002).

[5] A. V. Glushkov et al., JETP Lett. 85, 131 (2007).

[6] A. V. Glushkov et al., Phys. Rev. D82, 041101 (2010).

[7] M. Risse et al., Phys. Rev. Lett. 95, 171102 (2005).

[8] G. I. Rubtsov et al., Phys. Rev. D 73, 063009 (2006).

[9] J. Abraham et al. [Pierre Auger Collaboration], Astropart. Phys. 27, 155 (2007).

[10] J. Abraham et al. [Pierre Auger Collaboration], Astropart. Phys. 29, 243 (2008).

[11] A. Aab et al. [Pierre Auger Collaboration], JCAP 1704 (2017) 009.

[12] T. Abu-Zayyad et al. [Telescope Array Collaboration], Phys. Rev. D 88, 112005 (2013).

[13] G. I. Rubtsov et al. [Telescope Array Collaboration], PoS ICRC 2015, 331 (2016).

[14] A. Aab et al. [Pierre Auger Collaboration], Astrophys. J. 789, 160 (2014).

[15] V. Berezinsky, P. Blasi and A. Vilenkin, Phys. Rev. D 58, 103515 (1998).

[16] K. Griesen, Phys. Rev. Lett. 16, 748 (1966)

[17] Z. T. Zatsepin and V. A. Kuz'min, Zh. Eksp. Teor. Fiz. Pis'ma Red. 4, 144 (1966)

[18] S. R. Coleman and S. L. Glashow, Phys. Rev. D 59, 116008 (1999).

[19] M. Galaverni and G. Sigl, Phys. Rev. Lett. 100, 021102 (2008).

[20] L. Maccione, S. Liberati and G. Sigl, Phys. Rev. Lett. 105, 021101 (2010).

[21] G. Rubtsov, P. Satunin and S. Sibiryakov, Phys. Rev. D 86, 085012 (2012).

[22] G. Rubtsov, P. Satunin and S. Sibiryakov, Phys. Rev. D 89 (2014) 123011.

[23] D. S. Gorbunov et al., JETP Lett. 80, 145 (2004).

[24] R. U. Abbasi et al. [HiRes Collaboration], Astrophys. J. 636, 680 (2006).

[25] M. Fairbairn, T. Rashba and S. Troitsky, arXiv:0901.4085 [astro-ph.HE].

[26] O. E. Kalashev, V. A. Kuzmin, D. V. Semikoz et al., Ultrahigh-energy neutrino fluxes and their constraints, Phys. Rev. D66 (2002) 063004

[27] R. Abbasi et al. [HiRes Collaboration], Astrophys. J. 684, (2008) 790.

[28] P. Abreu et al. [Pierre Auger Collaboration], Phys. Rev. D 84, (2011) 122005.

[29] P. Abreu et al. [Pierre Auger Collaboration], Astrophys. J. 755 (2012) L4.

[30] A. Aab et al. [Pierre Auger Collaboration], Phys. Rev. D 91 (2015) 092008.

[31] M. G. Aartsen et al. [IceCube Collaboration], Phys. Rev. Lett. 117 (2016) 241101.

[32] P.W. Gorham et al. [ANITA Collaboration], Phys. Rev. D 85, (2012) 049901(E). 
[33] I. Kravchenko et al. [RICE Collaboration], Phys. Rev. D 85, (2012) 062004.

[34] T.R. Jaeger, R.L. Mutel, K.G. Gayley [RESUN Collab.], Astropart. Phys. 34, (2010) 293.

[35] O. Scholten et al., Phys. Rev. Lett. 103, (2009) 191301.

[36] C.W. James et al. [LUNASKA Collaboration], Phys. Rev. D 81, (2010) 042003.

[37] J.D. Bray et al. Phys. Rev. D 91, (2015) 063002.

[38] T. Karg et al. [IceCube and Pierre Auger and Telescope Array Collaborations], JPS Conf. Proc. 9 (2016) 010021.

[39] M. G. Aartsen et al. [IceCube Collaboration], Phys. Rev. Lett. 113, 101101 (2014).

[40] T. Abu-Zayyad, R. Aida, M. Allen, R. Anderson, R. Azuma, E. Barcikowski, J. W. Belz and D. R. Bergman et al., Astrophys. J. 768, L1 (2013).

[41] M. Risse and P. Homola, Mod. Phys. Lett. A 22, 749 (2007).

[42] O. E. Kalashev, G. I. Rubtsov, S. V. Troitsky, Phys. Rev. D80, 103006 (2009).

[43] D. Heck et al., Report FZKA-6019 (1998), Forschungszentrum Karlsruhe.

[44] W. R. Nelson, H. Hirayama, D.W.O. Rogers, SLAC-0265.

[45] P. Homola et al., Comp. Phys. Comm. 173 (2005) 71.

[46] S. Ostapchenko, Nucl. Phys. Proc. Suppl. 151, 143 (2006).

[47] A. Ferrari et al., CERN-2005-010; A. Fasso, A. Ferrari, J. Ranft et al., eConf C0303241, MOMT005 (2003).

[48] B. Stokes et al., Proc. of the International Symposium on UHECR, Nagoya, December 10-12, (2010).

[49] S. Agostinelli et al. [GEANT4 Collaboration], Nucl. Instrum. Meth. A 506, 250 (2003).

[50] R.U. Abbasi et al. [Telescope Array Collaboration], Phys. Lett. A 381, 2565 (2017).

[51] J. Abraham et al. [Pierre Auger Collaboration], Phys. Rev. Lett. 100, 211101 (2008).

[52] G. Rubtsov, S. Troitsky for the Telescope Array Collaboration, J. Phys. Conf. Ser. 608, 012067 (2015).

[53] G. Ros, A. D. Supanitsky, G. A. Medina-Tanco, L. del Peral and M. D. Rodriguez-Frias, Astropart. Phys. 47, 10 (2013).

[54] Hoecker A, Speckmayer P, Stelzer J, Therhaag J, von Toerne E and Voss H 2007 PoS ACAT 040,2007 preprint arXiv:physics/0703039

[55] G. J. Feldman and R. D. Cousins, Phys. Rev. D 57 (1998) 3873.

[56] http://www.vaisala.com/en/products/

thunderstormandlightningdetectionsystems/Pages/NLDN. aspx 